\title{
Effect of endothelin and endothelin receptor blockade on capillary permeability in experimental pancreatitis
}

\author{
G Eibl, H G Hotz, J Faulhaber, M Kirchengast, H J Buhr, Th Foitzik
}

\begin{abstract}
Background-Capillary leakage with fluid loss into the third space contributes to many of the early systemic complications in severe acute pancreatitis. There has been increasing interest in endothelin as one of the factors affecting capillary permeability.

Aim-To elucidate further the role of endothelin in the development of capillary leakage in acute pancreatitis by investigating the effect of exogenous endothelin administration and endothelin receptor blockade in sham operated animals and two models of acute pancreatitis.

Methods-Determination of capillary permeability in the pancreas and colonic mucosa by quantifying extravasation of fluorescein labelled dextran using a novel computer assisted video image analysis system.
\end{abstract}

Results-Pancreatic and colonic capillary permeability increased stepwise from mild to severe acute pancreatitis. Endothelin increased pancreatic and colonic capillary permeability in healthy animals and animals with mild acute pancreatitis but had no additional adverse effect in severe acute pancreatitis. Endothelin receptor blockade decreased pancreatic capillary permeability in sham operated rats but had no effect on the colon. In mild and severe acute pancreatitis, endothelin receptor blockade stabilised increased capillary permeability in both the pancreas and colon.

Conclusions-Endothelin plays an important role in mediating capillary permeability in the pancreas. In severe pancreatitis, it increases capillary permeability even outside the pancreas, thereby contributing to capillary leakage. Endothelin receptor blockade significantly reduces capillary permeability in acute pancreatitis both in and outside the pancreas, suggesting a therapeutic approach to counteract capillary leakage in severe acute pancreatitis. (Gut 2000;46:390-394)

Keywords: pancreatitis; endothelin; endothelin receptor antagonist; microcirculation; capillary permeability; fluid sequestration

Dr Th Foitzik, Department of Surgery, Benjamin

Franklin Medical Centre,

Freie Universität Berlin,

Hindenburgdamm 30,

D-12200 Berlin, Germany

Accepted for publication 5 October 1999
Acute pancreatitis, a disease with high morbidity and mortality, can only be managed by supportive treatment. Early pathogenic events involve premature intrapancreatic protease activation and acinar cell injury causing a local and systemic inflammatory response mediated by (pro)inflammatory cytokines and secondary mediators. Recent research has suggested that the degree to which these mediators escape into the circulation correlates significantly with disease severity. ${ }^{1-4}$ Disease severity not only comprises local tissue injury but also (maybe even more importantly) the systemic sequelae associated with systemic inflammatory response syndrome. Increased capillary permeability with subsequent fluid loss from the intravascular compartment into the third space is believed to contribute substantially to these systemic sequelae. ${ }^{5}$ Therefore it is important to identify the dominant vasoactive mediators that increase capillary permeability. Vasoactive substances assumed to be involved include platelet activating factor, nitric oxide, bradykinin, prostaglandins, and endothelin..$^{6-9}$ In the light of previous findings showing that endothelin receptor blockade ameliorates disease severity, ${ }^{5}$ this study aimed to evaluate further how capillary permeability is affected by endothelin-1, the most abundant and potent member of the endothelin family. Our underlying questions were as follows. (a) Does exogenous endothelin-1 increase capillary permeability in healthy rats? (b) Is the pancreas especially susceptible to endothelin-1, or are other organs similarly affected? (c) Does exogenous endothelin-1 likewise increase capillary permeability in mild (associated with a normal endogenous endothelin-1 level and normal or slightly increased capillary blood flow and permeability) and severe (increased endogenous endothelin-1, decreased capillary blood flow, and increased permeability) acute pancreatitis? (d) How does endothelin receptor blockade (endothelin-1 receptor antagonist) influence capillary permeability in and outside the pancreas in healthy rats and in those with mild and severe acute pancreatitis?

\section{Methods}

ANIMAL PREPARATION AND INDUCTION OF PANCREATITIS

All experiments were conducted in accordance with the national guidelines for the use and care of laboratory animals and were approved by the local ethics committee. After overnight fasting, male Sprague-Dawley rats (mean (SD) weight $330(20) \mathrm{g})$ were anaesthetised with intraperitoneal pentobarbital $(20 \mathrm{mg} / \mathrm{kg})$ and ketamine $(40 \mathrm{mg} / \mathrm{kg})$. Polyethylene catheters (internal diameter $0.5 \mathrm{~mm}$ ) were inserted into 
both jugular veins and the left carotid artery, subcutaneously tunnelled to the neck, and advanced through a steel tether, which allowed blood sampling and intravenous access in the unrestrained animals.

Mild oedematous acute pancreatitis was induced by a continuous intravenous infusion of $5 \mu \mathrm{g} / \mathrm{kg} / \mathrm{h}$ cerulein (Farmitalia, Freiburg, Germany) over six hours, and severe necrotising acute pancreatitis by cerulein hyperstimulation superimposed on a standardised retrograde infusion of $0.5 \mathrm{ml}$ of $10 \mathrm{mmol} / 1$ glycodeoxycholic acid (Sigma, St Louis, Missouri, USA) into the biliopancreatic duct for 10 minutes. As previously described, this technique requires mini-laparotomy for canulation of the biliopancreatic duct by puncturing the duodenum. ${ }^{10}$ For comparison, laparotomy and canulation of the biliopancreatic duct was likewise performed in animals with mild pancreatitis (intravenous cerulein, intraductal saline) and healthy control animals (intravenous and intraductal saline infusion). As an improvement on the original technique, ${ }^{10}$ a special infusion pump (IVAC 770; Lilly Medisintechnik, Giessen, Germany) was used for the pressure controlled (30 $\mathrm{mm} \mathrm{Hg}$ ), volume controlled $\left(0.5 \mathrm{~cm}^{3}\right)$, and time controlled (10 minutes) intraductal glycodeoxycholic acid infusion.

STUDY PROTOCOL AND TREATMENT

Six hours after sham operation ( $\mathrm{n}=30$ rats; group 1$)$ or induction of mild ( $n=30$; group 2$)$ or severe ( $n=45$; group 3 ) acute pancreatitis, animals were randomised into three groups for intravenous injection of normal saline $0.9 \%$ (A), $1.25 \mu \mathrm{g} / \mathrm{kg} / \mathrm{h}$ endothelin-1 (B; provided by Knoll AG, Ludwigshafen, Germany), or 50 $\mathrm{mg} / \mathrm{kg}$ of the endothelin-1 receptor antagonist LU-135252 (C; provided by Knoll AG).

Systemic cardiorespiratory variables were monitored over the next six hours. The animals were subsequently relaparotomised for intravital microscopic determination of capillary permeability.

DETERMINATION OF CAPILLARY PERMEABILITY Capillary permeability was determined by intravital microscopy using the same technique and equipment as in previous studies. ${ }^{11} 12$ Briefly, the abdomen was opened by a small midline incision. Either the duodenum together with the head of the pancreas or the ascending colon were mobilised and placed on a special stage for intravital microscopy. After a 10 minute stabilisation period, three randomly chosen regions $(400 \times 325 \mu \mathrm{m})$ in the head of the pancreas or the colonic mucosa (after antimesenteric enterotomy ${ }^{13}$ ) were recorded. Subsequently $0.2 \mathrm{ml} 5 \%$ fluorescein isothiocyanate labelled dextran (molecular mass 150000 Da; Sigma, Deisenhofen, Germany) were injected, and, after 30 minutes, the same three regions were recorded for off line determination of capillary permeability using a commercially available video image analysis system (Cap-Image, V.6.01; Zeintl, Heidelberg, Germany). This computer assisted system quantifies the increase in perivascular fluorescein intensity resulting from the extravasation of the fluorescence labelled dextran over time (here 30 minutes).

The total time needed for the exposure and recording of the microcirculatory beds was 60-90 minutes per animal. Heart rate, systolic and mean arterial pressure, and blood gases were measured before and after intravital microscopy. We only included data from animals in stable cardiorespiratory condition for analysis of the microcirculatory variables to avoid bias possibly resulting from systemic cardiorespiratory derangement. Exclusion criteria were: mean arterial pressure $<80 \mathrm{~mm} \mathrm{Hg} ; \mathrm{PO}_{2}<80 \mathrm{~mm} \mathrm{Hg}$; $\mathrm{PCO}_{2}>50 \mathrm{~mm} \mathrm{Hg} ; \mathrm{pH}<7.3$ or $>7.5$.

\section{STATISTICAL ANALYSIS}

All results are expressed as mean (SEM). Variables were tested for group differences with Student's $t$ test and analysis of variance as appropriate. $\mathrm{p}<0.05$ was considered to be significant.

\section{Results}

\section{SHAM OPERATED ANIMALS}

Endothelin-1 administration (group 1B) had no measurable effect on the systemic cardiorespiratory variables except to produce an immediate transient decrease in systolic arterial blood pressure (10-30 $\mathrm{mm} \mathrm{Hg}$ ) which normalised within two to three minutes, followed by an increase in arterial blood pressure $(30-40 \mathrm{~mm}$ $\mathrm{Hg}$ ), which returned to baseline within 10-15 minutes. Blood pressure at later time points was not different from sham operated animals given saline (group 1A). An increase in packed cell volume developed in all endothelin-1 treated animals (baseline 48 (1) $v 54$ (1) six hours after the start of endothelin-1 administration). Capillary permeability increased by $104 \%$ in the pancreas (fig 1A) and $82 \%$ in the colon (fig 1B). Endothelin-1 receptor antagonist (group 1C) had no significant effect on packed cell volume and cardiorespiratory variables; capillary
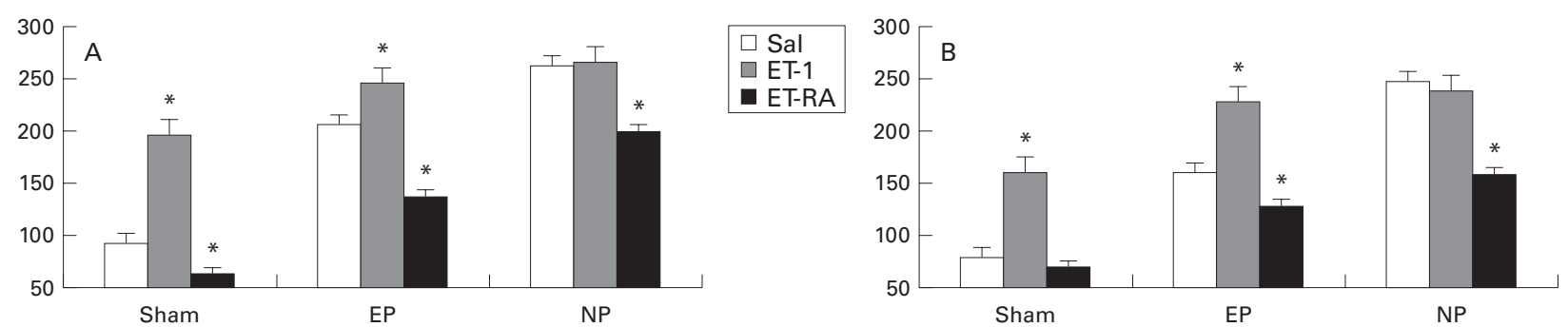

Figure 1 Effect of endothelin-1 (ET-1) and endothelin receptor antagonist (ET-RA) on capillary permeability (measured as the increase in perivascular density (\%) 30 minutes after injection of fluorescein isothiocyanate labelled dextran 150) in the pancreas (A) and colon (B) of sham operated animals and animals with mild (EP) or severe (NP) acute pancreatitis. ${ }_{p}^{*}<0.05$ compared with saline injected animals (Sal). 
permeability decreased in the pancreas (fig 1A) but was not significantly affected in the colon (fig 1B).

\section{MILD PANCREATITIS}

Compared with saline treated sham operated controls (group 1A), animals with oedematous acute pancreatitis given saline (group 2A) showed an increase in packed cell colume (48 (1) $v 51(1))$ and capillary permeability in both the pancreas and colon (fig 2). This increase was more pronounced in the pancreas than in the colon (114\% increase in the pancreas compared with $82 \%$ increase in the colon). Cardiorespiratory variables remained unchanged. Endothelin-1 (group 2B) further increased packed cell volume (51 (1) v 55 (1)) and significantly increased capillary permeability by $40 \%$ in the pancreas and $69 \%$ in the colon (fig 1). Endothelin-1 receptor antagonist (group 2C) significantly reduced packed cell volume (51 (1) $v 47$ (1)) and capillary permeability by $69 \%$ in the pancreas (fig $1 \mathrm{~A}$ ) and $33 \%$ in the colon (fig 1B). Cardiorespiratory variables did not differ significantly between the treatment groups.

\section{SEVERE PANCREATITIS}

All animals in which severe acute pancreatitis was induced developed significant haemoconcentration (packed cell volume 56 (1)) and showed an increase in heart rate and decrease in mean arterial pressure (compared with sham operated animals and animals with mild acute pancreatitis at six hours). However, no animal had to be excluded at this point according to the defined criteria. After treatment, four animals treated with saline developed cardiorespiratory insufficiency and died before intravital microscopy. Five animals given endothelin- 1 and two animals treated with endothelin-1 receptor antagonist died before intravital microscopic determination of capillary permeability. All other animals were in stable cardiorespiratory condition at the time of intravital microscopy. There were no significant differences in cardiorespiratory variables between treatment groups. Packed cell volume, however, was significantly reduced in animals treated with the endothelin receptor antagonist (51 (1) $v 56$ (1)). Administration of endothelin did not further increase the packed cell volume. Two animals (one treated with saline, one treated with endothelin) that survived intravital microscopy could not be included in the final analysis of capillary permeability, because the final assessment of arterial blood gases indicated severe respiratory insufficiency (see exclusion criteria). Compared with

Figure 2 Capillary permeability (measured as the increase in perivascular density (\%) 30 minutes after injection of fluorescein isothiocyanate labelled dextran 150) in the pancreas and colon of untreated sham operated animals (no acute pancreatitis) and animals with mild (EP) or severe (NP) acute pancreatitis. ${ }_{p}<0.05 v$ sham operated: $t p<0.05 \cup E P$.

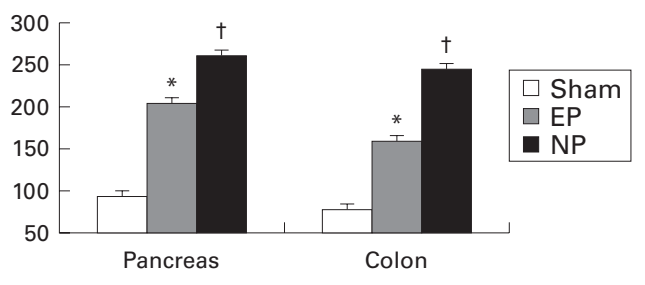

untreated sham operated animals and untreated animals with mild pancreatitis, pancreatic and colonic capillary permeability in rats with severe pancreatitis given saline were significantly increased $(248$ (17)\% in the colon and $284(16) \%$ in the pancreas) (fig 2). Endothelin administration did not further increase capillary permeability in the pancreas and colon (fig 1A,B). However, endothelin receptor antagonist decreased capillary permeability in the pancreas by $63 \%$ (fig 1A) and in the colon by $90 \%$ (fig $1 \mathrm{~B}$ ).

\section{Discussion}

Increased capillary permeability leading to fluid loss from the intravascular space and fluid sequestration into the third space is a hallmark of severe acute pancreatitis. Clinically, capillary leakage is reflected by intravascular fluid loss leading to hypovolaemia (low central venous pressure), haemoconcentration (high packed cell volume), and extravascular fluid sequestration - for example, in the retroperitoneum, lungs, and pleural and abdominal cavities. Factors believed to increase capillary permeability in severe pancreatitis include platelet activating factor, nitric oxide, bradykinin, prostanoids, and endothelin..$^{6-9} 14$ In this study, we report our findings with endothelin-1 and endothelin A receptor blockade (endothelin-1 receptor antagonist), which we chose on the basis of previous investigations showing that endothelin-1 aggravates acute pancreatitis whereas endothelin-1 receptor antagonist improves survival considerably. ${ }^{5}$ Measurements of packed cell volume and extravascular fluid sequestration implied that these effects were associated with the stablisation of increased capillary permeability. Therefore this study aimed to evaluate further the effect of endothelin-1 and a specific endothelin-1 antagonist (LU-135252) on capillary permeability in two different organ beds (pancreas and colon) of two acute pancreatitis models using direct intravital fluorescence microscopy and a novel computer assisted video image analysis system for quantitative assessment.

\section{METHODOLOGICAL CONSIDERATIONS}

The two models of acute pancreatitis (mild/ oedematous and severe/necrotising) have been extensively characterised histologically as well as by biochemical variables, systemic disease sequelae (including changes in fluid homoeostasis), and microcirculatory variables. ${ }^{10}{ }^{15}$ Intravital microscopy and quantification of capillary permeability by Cap-Image are also well established in our laboratory. As continuous extravasation of fluorescein isothiocyanate labelled dextran causes brightness of the microscopic picture, which compromises further measurements, capillary permeability could only be determined in one organ at a time. We therefore used five animals per group to determine capillary permeability in the pancreas and also in the colonic mucosa. The latter was chosen as a major site of fluid extravasation in capillary leakage syndrome because of its extensive surface and capillary density.

The endothelin-1 dose was chosen according to the manufacturer's recommendations, 
based on large scale experiments with this agent in small rodents ${ }^{16}$ as well as on previous tests in our laboratory showing that doses lower than $0.5 \mu \mathrm{g} / \mathrm{kg}$ have no effect on blood pressure or microcirculatory variables, whereas doses higher than $1.5 \mu \mathrm{g} / \mathrm{kg}$ cause pronounced changes in both macrohaemodynamic variables and microcirculation. The dose of 1.25 $\mu \mathrm{g} / \mathrm{kg}$ was finally chosen because it was the highest dose that had no appreciable effects on systemic cardiorespiratory variables except to cause an immediate transient fall in systolic arterial blood pressure followed by a transient increase in arterial blood pressure (see above). This intermittent depressor effect, also observed by other investigators, ${ }^{17-20}$ is thought to result from the initial activation of nonselective endothelin B2 receptors located on the intraluminal side of endothelial cells mediating vasodilatation. This effect, however, seems to be immediately counteracted by either rapid degradation of intraluminal endothelin-1 or a compensatory pressor response when endothelin-1 reaches the high affinity endothelin A receptors on the vascular smooth muscle cells.

Plasma endothelin-1 levels were not measured in this experiment because it is generally accepted that it elicits its major effects in a paracrine manner, while endoluminal endothelin-1 is rapidly degraded. ${ }^{17}{ }^{21}$ In a previous study, we found significantly elevated plasma endothelin-1 levels in the severe model but not in mild oedematous acute pancreatitis. ${ }^{5}$

Depending on the dose, LU-135252 first blocks endothelin A receptors mainly found on vascular smooth muscle cells, which selectively bind endothelin-1 over endothelin-2 and endothelin-3 to mediate contraction. In higher doses, it also blocks the endothelin $\mathrm{B}$ receptors mediating dilatation. Details on the properties of LU-135252 have been reported elsewhere. ${ }^{1622}{ }^{23}$ We chose a dose of $50 \mathrm{mg} / \mathrm{kg}$ for the present experiments, because doses as high as $100 \mathrm{mg} / \mathrm{kg}$ are well tolerated in rats and do not cause any appreciable changes in systemic haemodynamic variables (as shown in previous experiments ${ }^{5}$ ). This dose has been shown to block endothelin-A receptors selectively (suggesting that the effect of endothelin-1 on capillary permeability is primarily mediated by these receptors). This also agrees with the findings of other groups showing the inhibition of endothelin-1 induced plasma volume loss and reduced extravasation of Evans blue dye after treatment with other endothelin A receptor blockers ${ }^{24}$ and with recent studies of our group showing that endothelin A but not endothelin B receptor blockade mediates capillary leakage in severe acute pancreatitis. ${ }^{25}$ This also explains the different results obtained with (non-selective) endothelin antagonists.

INTERPRETATION OF RESULTS

Incremental extravasation of fluorescein isothiocyanate labelled dextran in the pancreas from mild to severe acute pancreatitis indicates that the increase in capillary permeability correlates with the magnitude of local tissue injury. As there is no evidence from the literature or our own histological studies that the capillaries are injured, functional rather than morphological changes in the endothelium probably cause plasma extravasation. Although capillary permeability was significantly higher in severe acute pancreatitis, pancreatic oedema was similar or even more pronounced in mild acute pancreatitis (as scored histologically ${ }^{5}$ ). This can be explained by the observation that the increase in capillary permeability is mainly confined to the pancreas in mild acute pancreatitis, whereas fluid also extravasates into the colon (and probably other capillary beds) in severe acute pancreatitis. Another reason for increased fluid loss from the pancreas in oedematous acute pancreatitis may be capillary blood flow, which is increased in mild acute pancreatitis but decreased in severe acute pancreatitis. ${ }^{15}$ The fact that the increase in capillary permeability was more pronounced in the pancreas than in the colon in mild acute pancreatitis agrees with the observation that the disease process is largely confined to the pancreas: neither animals nor patients with mild acute pancreatitis develop significant systemic disease sequelae or complications. In contrast, extremely increased colonic capillary permeability in severe acute pancreatitis seems to reflect the systemic reactions discussed above.

The endothelin-1 induced increase in capillary permeability observed in otherwise healthy sham operated rats suggests that endothelin-1 also plays a role in mediating capillary permeability in other conditions such as trauma. This also agrees with other studies emphasising the effect of endothelin on vascular permeability in various organs and tissues in conjunction with diseases and possibly under physiological conditions as well. ${ }^{242627}$ Failure of endothelin to increase capillary permeability further in severe acute pancreatitis indicates that all endothelin receptors were already saturated by endogenous endothelin-1 released in the course of severe acute pancreatitis. This is in accordance with our previous findings that endothelin-1 in plasma is significantly increased in severe acute pancreatitis (but normal in mild acute pancreatitis). ${ }^{5}$ The fact that endothelin- 1 has a dose dependent effect and is only harmful at higher concentrations ${ }^{28}$ would also explain the contradictory results observed with various doses of (exogenous) endothelin-1 in different models of acute pancreatitis (with or without increased endogenous endothelin-1 levels or pre-existing microcirculatory disorders).$^{29}$

It is still unclear how endothelin-1 affects vascular permeability and whether this effect is associated with its well known action as a vasoconstrictor of precapillary and postcapillary vessels and venules. ${ }^{30}$ An endothelin-1 associated increase in postcapillary resistance may elevate the hydrostatic pressure in the capillary bed, thereby enhancing fluid transfer from the intra- to the extra-vascular space. Alternatively, it is conceivable that endothelin-1 affects capillary permeability by regulating interendothelial gaps through the contraction of vascular elements such as myosin and actin contained in endothelial cells and surrounding pericytes. ${ }^{31} 32$ 
The endothelin-1 receptor antagonist induced decrease in capillary permeability observed in the pancreas but not in the colon of otherwise healthy sham operated rats suggests that endogenous endothelin-1 was released during organ exposure for intravital microscopy and that the pancreas was more susceptible to this manipulation, endothelin itself or both. Several investigators have previously shown that the pancreas is especially susceptible to endothelin-1. For example, exogenous endothelin-1 in doses not affecting systemic haemodynamic variables significantly reduced pancreatic perfusion whereas other organsfor example, heart, lungs, liver-were not affected or even showed increased blood flow. ${ }^{33}$ This was explained by different expression of endothelin receptors.

Capillary permeability in acute pancreatitis after endothelin-1 receptor antagonist treatment did not reach the values measured in healthy animals, which indicates that endothelin-1 is not the only mediator of capillary permeability in acute pancreatitis. This underlines the need to study further the effects of other vasoactive substances and mediators on capillary permeability in future experiments.

In summary, these results answer our questions as follows. (a) There is a stepwise increase in capillary permeability from mild to severe acute pancreatitis. (b) Exogenous endothelin-1 increases capillary permeability in animals without pancreatitis and those with mild acute pancreatitis. (c) The endothelin-1 induced increase in capillary permeability is more pronounced in the pancreas than in the colon (suggesting that the pancreas is especially susceptible to endothelin). (d) Exogenous endothelin-1 does not further increase capillary permeability in severe acute pancreatitis (suggesting that all endothelin-1 receptors are already occupied by endogenous endothelin-1). (f) Endothelin-1 receptor antagonist reduces (increased) capillary permeability in mild and severe acute pancreatitis in and outside the pancreas. $(g)$ In sham operated animals, only pancreatic capillary permeability is affected (suggesting an important role for endothelin-1 in regulating pancreatic microcirculation).

In conclusion, these results underline the role of the endothelin system in mediating capillary permeability in acute pancreatitis and suggest that endothelin-1 blockade by specific endothelin receptor antagonists may become a powerful tool in the treatment of acute pancreatitis because they stabilise increased capillary permeability which contributes to many of the systemic sequelae in this disease.

This work was presented in part at the European Pancreas Club, Thessaloniki, Greece, June 10-13, 1998.

1 Kingsnorth A. Role of cytokines and their inhibitors in acute pancreatitis. Gut 1997;40:1-4

2 Gross V, Leser HG, Heinisch A, et al. Inflammator mediators and cytokines. New aspects of the pathophysiology and assessment of severity of acute pancreatitis? Hepato-gastroenterol 1993;40:522-30.

3 De Beaux AC, Goldie AS, Ross JA, et al. Serum concentrations of inflammatory mediators related to organ failure in patients with acute pancreatitis. Br F Surg 1996;83:349-53.

4 Heath DI, Cruickshank A, Gudgeon AM, et al. The relationship between pancreatic enzyme release and activa- tion and the acute-phase protein response in patients with acute pancreatitis. Pancreas 1995; 10:347-53.

5 Foitzik T, Faulhaber J, Hotz HG, et al. Endothelin receptor blockade improves fluid sequestration, pancreatic capillary blood flow and survival in severe experimental pancreatitis. Ann Surg 1998;228:670-5.

6 Yamanaka K, Saluja AK, Brown GE, et al. Protective effects of prostaglandin E1 on acute lung injury of caeruleininduced acute pancreatitis in rats. Am f Physiol 1997;272. G23-30.

7 Weidenbach H, Lerch MM, Gress TM, et al. Vasoactive mediators and the progression from edematous to necrotizing experimental acute pancreatitis. Gut 1995;37:434-40.

8 Dobosz M, Hac S, Wajda Z. Does nitric oxide protect from microcirculatory disturbances in experimental acute pancreatitis in rats? Int 7 Microcirc Clin Exp 1996;16:221-6.

9 Yotsumoto F, Manabe T, Ohshio G, et al. Role of pancreatic blood flow and vasoactive substances in the development of canine acute pancreatitis. $\mathcal{F}$ Surg Res 1993;55:531-6.

10 Schmidt J, Rattner DW, Lewandrowski K, et al. A better model of acute pancreatitis for evaluating therapy. Ann Surg 1992;215:44-56.

11 Mithöfer K, Schmidt J, Gebhard MM, et al. Measurement of blood flow in pancreatic exchange capillaries with
FITC-labelled erythrocytes. Microvasc Res 1995;49:33-48.

12 Hotz HG, Schmidt J, Ryschich EW, et al. Isovolemic hemodilution with dextran prevents contrast medium induced impairment of pancreatic microcirculation in necrotizing pancreatitis in the rat. Am f Surg 1995;169: $161-6$.

13 Hotz HG, Foitzik T, Rohweder J, et al. Intestinal microcirculation and gut permeability in acute pancreatitis. Early changes and therapeutic implications. $\mathcal{f}$ Gastrointest Surg 1998;2:518-25.

14 Bloechle C, Kusterer K, Kuehn RM, et al. Inhibition of bradykinin $\mathrm{B} 2$ receptor preserves microcirculation in experimental pancreatitis in rats. Am $\mathcal{F}$ Physiol 1998;274: G42-51.

15 Klar E, Schratt W, Foitzik T, et al. Impact of microcirculatory flow pattern changes on the development of acute edematous and necrotizing pancreatitis in rabbit pancreas. Dig Dis Sci 1994;39:2639-44.

16 Knoll. Investigator brochure LU-135252. 2nd ed. Ludwigshafen: Knoll, 1997:14-32.

17 Mateo AO, De Artinano AA. Highlights on endothelins: a review. Pharmacol Res 1997;36:329-35.

18 Lippton H, Goff J, Hyman A. Effects of endothelin in the systemic and renal vascular beds in vivo. Eur $\mathcal{F}$ Pharmacol 1988;155:197-9.

19 Wright CE, Fozard JR. Regional vasodilatation is a prominent feature of the hemodynamic response to endothelin in anesthetized spontaneously hypertensive rats. Eur F Pharmacol 1988;155:201-3.

20 Winquist RJ, Bunting PB, Garsky VM, et al. Prominent depressor response to endothelin in spontaneously hypertensive rats. Eur F Pharamcol 1989;163:199-203.

21 Wagner OF, Christ G, Wojta J, et al. Polar secretion of endothelin-1 by cultured endothelial cells. $\mathcal{F}$ Biol Chem 1992;267:16066-8.

22 Raschak M, Unger L, Riechers H, et al. Receptor selectivity of endothelin antagonists and prevention of vasoconstriction and endothelin-induced sudden death. $f$ Cardiovasc Pharmacol 1995;26(suppl 3):S397-9.

23 Riechers H, Albrecht H-P, Amberg W, et al. Discovery and optimization of a novel class of orally active nonpeptidic endothelin-A receptor antagonists. F Med Chem 1996;39: 2123-8.

24 Filep JG, Sirois MG, Földes-Filep E, et al. Enhancement by endothelin-1 of microvascular permeability via the activation of $\mathrm{ET}_{\mathrm{A}}$ receptors. Br F Pharmacol 1993;109:880-6.

25 Foitzik T, Eibl G, Forgacs B, et al. Therapy of microcirculatory disorders in severe acute pancreatitis. Endothelin-A but not endothelin-B receptor blockade reduces capillary leakage. Pancreas 1998;17:433.

26 Filep JG, Sirois MG, Rousseau A, et al. Effects of endothelin-1 on vascular permeability in the conscious rat: interactions with platelet-activating factor. $\mathrm{Br} \mathcal{F}$ Pharmacol 1991;104:797-804.

27 Dahlöf B, Gustafsson D, Hedner T, et al. Regional hemodynamic effects of endothelin-1 in rat and man: unexpected adverse reactions. F Hypertens 1990;8:811-17.

28 Brain SD, Crossman DC, Buckley TL, et al. Endothelin-1: demonstration of potent effects of the microcirculation of humans and other species. I Cardiovasc Pharmacol 1989;13(suppl 5):S147-9.

29 Kogire M, Inoue K, Higashide SI, et al. Protective effects of endothelin-1 on acute pancreatitis in rats. Dig Dis Sci 1995; 40:1207-12.

30 Yang ZH, Bühler FR, Diederich D, et al. Different effects of endothelin-1 on CAMP- and cGMP-mediated vascular relaxation in human arteries and veins: comparison with norepinephrine. 7 Cardiovasc Pharmacol 1989;13(suppl 5):S129-31.

31 Becker CG, Nachman RL. Contractile proteins of endothelial cells, platelets and smooth muscles. Am F Pathol 1973; 71:1-22.

32 LeBeux YJ, Willemot J. Actin- and myosin-like filaments in rat brain pericytes. Anat Rec 1978;190:811-26.

33 Takaori K, Inoue K, Kogire M, et al. Effects of endothelin on microcirculation of the pancreas. Life Sci 1992;51:615- 